\title{
Plataforma PGuide: um modelo de recomendação para usuários móveis
}

\author{
Alezy Oliveira Lima ${ }^{1,2}$, Ricardo Alexandre Afonso ${ }^{2}$, Thiago Bruno Melo de Sales ${ }^{2}$ \\ ${ }^{1}$ Centro de Informática - Universidade Federal de Pernambuco (UFPE) \\ Av. Jornalista Anibal Fernandes, Cidade Universitária - 50740-560 - Recife - PE - Brasil \\ ${ }^{2}$ Campus Arapiraca - Universidade Federal de Alagoas (UFAL) \\ Av. Manoel Severino Barbosa, Bom Sucesso - 57309-005 - Arapiraca - AL - Brasil \\ aol@cin.ufpe.br, \{afonso055, thiagobrunoms\}@gmail.com
}

\begin{abstract}
Mobile devices as smartphones and tablet PC have become an important platform for communication and information access. This implies the emergence of new forms of interaction with these technologies, which include the use of mobile devices as a resource in support of decision making. Take into account the user context while the same search information to maximize their level of satisfaction with the system since the information spaceltime may provide further details on the process of information filtering. In this perspective, this works presents a recommender system able to customize products and services based on geographic location, as well as in estimated consumption patterns of mobile users.
\end{abstract}

Resumo. Dispositivos móveis tais como smartphones e tablets PC têm se tornado uma importante plataforma de comunicação e acesso à informação. Isto implica no surgimento de novas formas de interação com estas tecnologias, que incluem a utilização de dispositivos móveis como recurso no apoio à tomada de decisão. Considerar o contexto do usuário enquanto o mesmo busca informações deve maximizar seu grau de satisfação junto ao sistema, uma vez. que a informação espaço/tempo pode proporcionar maiores detalhes nos processos de filtragem de informação. Nesta perspectiva, é apresentado um sistema de recomendação capaz de personalizar ofertas de produtos e serviços com base na localização geográfica, bem como nas estimativas de padrões de consumo dos usuários móveis.

\section{Introdução}

Dispositivos como smartphones e tablets estão mais presentes no cotidiano das pessoas do que há poucos anos atrás [Ricci 2010] [Lima et al. 2012]. Isso é reflexo da clara migração das atividades antes realizadas nos computadores de mesa para a computação móvel e pervasiva [Weiser 1991], na qual o acesso à informação ocorre em qualquer lugar, a qualquer momento. A ampla expansão da computação móvel é influenciada por diversos fatores, dentre eles destacam-se os avanços das tecnologias de redes sem fio, tais como Wi-Fi e $3 \mathrm{G}$, o barateamento na produção dos dispositivos móveis, o crescente número de novos aplicativos voltados para plataformas móveis e, principalmente, a acirrada concorrência entre as fabricantes de dispositivos móveis.

Com efeito, várias aplicações buscam proporcionar serviços com o objetivo de facilitar atividades regulares ou mesmo proporcionar conforto aos seus usuários. Para o 
fornecimento de serviços e informações cada vez mais relevantes, várias técnicas baseadas em inteligência artificial podem ser empregadas. Dentre elas, destacam-se os Sistemas de Recomendação [Ricci et al. 2011], que são constituídos de técnicas que têm o objetivo de fornecer sugestões de itens que possam ser do interesse do usuário. Estas técnicas podem ser incorporadas aos mais variados tipos de sistemas de informação, em diversas plataformas, no intuito de oferecer produtos e serviços com maior precisão e probabilidade de aquisição por parte do usuário.

Os sistemas de recomendação têm sido adotados em várias ferramentas de software com o objetivo de incrementar seus serviços. Dentre elas destacam-se as ferramentas voltadas para o comércio eletrônico. Naturalmente é possível observar em lojas virtuais a sugestão de itens que estão correlacionados aos itens visitados pelo usuário, e isso tem impulsionado o sucesso do comércio eletrônico na era da informação.

As tecnologias de computação móvel, associadas aos sistemas de recomendação, podem potencializar ainda mais a qualidade dos serviços no comércio eletrônico, uma vez que experiências com novas grandezas associadas (por exemplo, o espaço e o tempo) podem beneficiar outros cenários e indicar resultados cada vez mais satisfatórios para o consumidor.

\subsection{Problemática e objetivos}

Dificuldades podem ser encontradas quando se deseja comprar algum produto (alimentício, vestuário etc) e não é possível determinar a melhor estratégia de compra com base em parâmetros como: qual loja oferece o melhor preço, melhor forma de pagamento e melhores promoções; ou qual loja está mais próxima a sua localização atual, buscando considerar custos como deslocamento e tempo. Essa tomada de decisão pode se tornar ainda mais complexa quando tais parâmetros devem ser combinados. Como exemplo, considere duas lojas A e B que disponibilizam um mesmo produto " $\mathrm{X}$ ". A loja "A" oferece preços promocionais para este produto, embora os custos do deslocamento de um usuário localizado em um ponto "P" até a loja "A" possam ser superiores em relação à economia alcançada com as promoções. Desta forma, talvez seja melhor recomendá-lo a loja "B".

Ou seja, como automatizar a tomada de decisão de um usuário móvel que deseja comprar uma unidade ou um conjunto de produtos, porém, precisa lidar com uma grande quantidade de lojas e opções de produtos disponíveis relativamente próximas a ele? Ainda, como realizar uma análise automatizada levando-se em consideração grandezas e critérios distintos entre si, tais como preço e distância?

Dessa forma, este trabalho objetiva desenvolver um sistema de recomendação baseado em técnicas de inteligência artificial que permita a realização de análises de ofertas de produtos e serviços, no intuito de oferecer ao usuário as melhores indicações de produtos e serviços. Isto será possível graças à análise individualizada do contexto no qual o mesmo está inserido, estimativa de perfil e padrões de consumo. Com isto, esperase obter:

- recomendação individualizada com o objetivo de auxiliar o consumidor que usa aplicações móveis no momento da tomada de decisão;

- descoberta automática de conhecimento através da análise do comportamento de usuários móveis;

- exposição de anúncios com uma maior probabilidade de aquisição com base na estimativa de padrões de consumo de cada usuário; 
- obtenção de um melhor custo-benefício para os anunciantes, visto que seus anúncios atingirão um público-alvo que tende a ser mais específico.

O trabalho está estruturado da seguinte maneira: na Seção 2 é apresentada a fundamentação teórica, delineando algumas técnicas e ferramentas utilizadas no desenvolvimento desse projeto. Na Seção 3 é apresentada uma revisão da bibliografia, onde são abordados alguns trabalhos relacionados a este projeto. Em seguida, está apresentada na Seção 4 a ferramenta proposta, através de seus diagramas, arquitetura, modelos e algoritmos de recomendação. Depois, na Seção 5 é apresentado um exemplo de aplicação para as ferramentas propostas. Finalmente, na Seção 6 são abordadas as considerações finais e a continuidade do projeto através de seus trabalhos futuros.

\section{Fundamentação Teórica}

\subsection{Sistemas de Recomendação}

Sistemas de Recomendação (RS) [Resnick and Varian 1997] são ferramentas e técnicas de software que fornecem sugestões de itens ${ }^{1}$ que podem ser úteis ao usuário [Ricci et al. 2011]. Estas sugestões podem estar relacionadas a vários processos de tomada de decisão, como a escolha de uma música, de um livro, de quais notícias podem ser relevantes ou quais itens que podem ser comprados em uma loja eletrônica.

Os RS foram desenvolvidos inicialmente a partir de situações típicas do cotidiano: as pessoas muitas vezes dependem de indicações fornecidas por outras pessoas em processos de tomada de decisão [Ricci et al. 2011]. O principal papel de RS no núcleo de um sistema computacional é prever a avaliação subjetiva que um usuário dará a um item no sistema, orientando e ajudando o mesmo localizar itens que ele poderá gostar [Schafer et al. 2001]. Esta previsão é calculada através de uma série de modelos que tenham uma característica em comum, isto é, explorando avaliações fornecidas pelo usuário para itens anteriormente visualizados ou adquiridos, ou mesmo seus similares [Ricci 2010].

Para atingir estes objetivos, uma vasta variedade de técnicas baseadas em RS foram propostas. Estas concentram-se em quatro principais: a filtragem colaborativa, a filtragem baseada em conteúdo, a filtragem baseada em conhecimento e a filtragem híbrida [Burke 2007].

Provavelmente a mais popular e consolidada [Burke 2002] entre as técnicas baseadas em RS, a filtragem colaborativa, em suas implementações mais simples, observa correlações entre usuários e suas avaliações sobre itens em um sistema de informação. O objetivo é prever a classificação de itens relevantes para um indivíduo baseando-se nas classificações fornecidas por outros indivíduos de características ou padrões similares [Ricci 2010].

$\mathrm{Na}$ filtragem baseada em conteúdo, os itens que serão recomendados para o usuário são selecionados através de suas características associadas (conjuntos de documentos e/ou descrições) [Lops et al. 2011]. Um RS baseado em conteúdo aprende os interesses de um usuário com base em características presentes em itens que o usuário tenha classificado anteriormente. Através deste aprendizado, é estabelecido um modelo ou perfil de interesses deste usuário para então realizar os processos de recomendação.

\footnotetext{
${ }^{1}$ O termo "item" é geralmente utilizado para caracterizar o objeto que o sistema recomenda ao usuário.
} 
Ainda, existe a técnica de filtragem baseada em conhecimento. Esta técnica permite o cálculo e recomendação de itens baseando-se no uso de estruturas de conhecimento que realizam inferências acerca das necessidades explícitas e preferências do usuário [Ricci 2010] [Burke 2007].

Por fim, as técnicas de filtragem híbrida são compostas pela combinação de duas ou mais técnicas com o objetivo de conquistar melhor performance, minimizando limitações de cada abordagem [Burke 2002]. Nestes casos onde são empregadas técnicas híbridas, normalmente a filtragem colaborativa é combinada com alguma outra técnica para se atingir objetivos específicos.

\subsection{Computação Móvel}

O crescimento extraordinário que tem ocorrido nos últimos anos nas áreas de comunicação, redes locais e serviços via satélite permite que informações e recursos possam ser acessados e utilizados em qualquer lugar, em qualquer momento [Mateus and Loureiro 2005]. A maior parte dos dispositivos modernos tem a capacidade de se comunicar com outros dispositivos, fixos e móveis, na rede. A este paradigma de computação, onde os dispositivos computacionais não necessariamente precisam ter uma posição fixa na rede, dá-se o nome de Computação Móvel.

A evolução da tecnologia favoreceu a popularização dos smartphones. Contudo, os sistemas operacionais proprietários ainda geravam uma série de dificuldades no desenvolvimento de aplicações para diferentes dispositivos [Euzébio 2011]. Por esta razão, um grupo de empresas lideradas pelo Google formou a Open Handset Alliance. Desta aliança surgiu o desenvolvimento do Android, um sistema operacional de código aberto voltado para dispositivos móveis.

Atualmente na liderança entre as plataformas móveis mais utilizadas no mercado, com cerca de 44,2\% do mercado norte-americano (conforme visto na Figura 1) e com aproximadamente 89 milhões de dispositivos vendidos (59\% do total comercializado) somente no primeiro trimestre de 2012 [IDC 2012], a plataforma Android pode ser considerada a mais popular entre os usuários móveis no mundo.

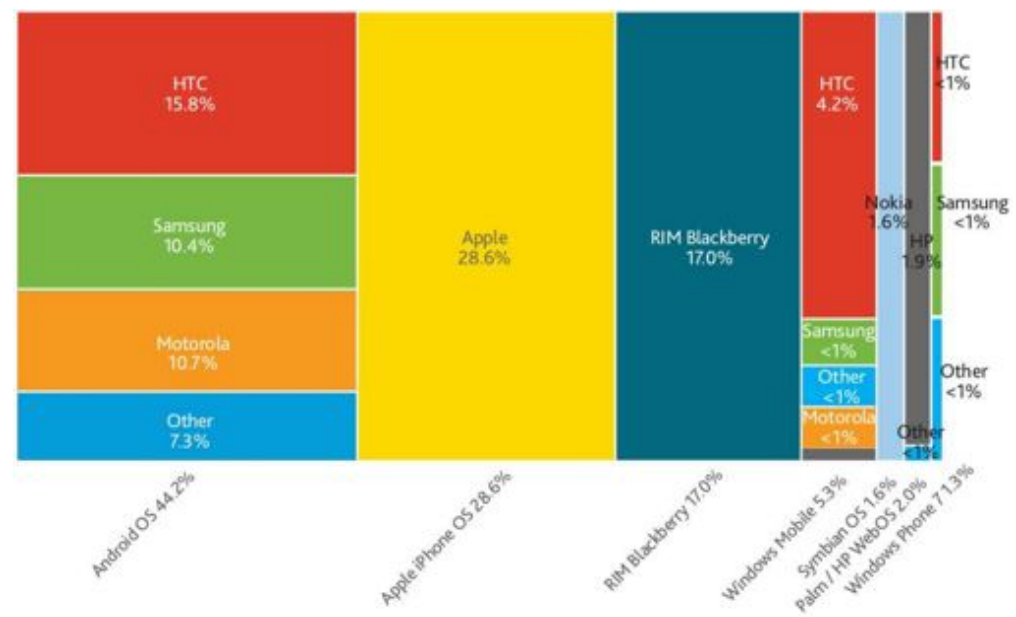

Figura 1. Divisão do domínio do mercado móvel. Fonte: [Nielsen Company 2011]. 


\section{Trabalhos Relacionados}

Em RS, percebe-se que boa parte dos esforços são dedicados no ramo do comércio eletrônico (ou e-commerce) e redes sociais. O primeiro exemplo encontrado na literatura consultada é o trabalho intitulado "Recommender Systems in ECommerce" [Schafer et al. 1999], onde é realizada uma análise da evolução da importância dos RS no e-commerce, atribuindo aos RS a responsabilidade pelo aumento na quantidade de vendas realizadas pelos sistemas. Para os autores, os RS são parte da personalização de um site, pois elas adaptam o mesmo às características pessoais de cada consumidor.

Em 2002, no trabalho “Travel Recommender Systems", [Ricci 2002] faz uma análise do uso de sistemas de recomendação em aplicações voltadas para viagens e turismo. Nele, o autor descreve que os RS emergiram inicialmente como ferramentas de filtragem de itens inapropriados para as necessidades dos usuários. Entretanto, com o tempo as pesquisas e os novos protótipos lançados mostraram que os papéis e as funções dos RS podem ser bem variados.

Ainda em 2002, em "Hybrid Recommender Systems: Surveys and Experiments”, [Burke 2002] realizou uma análise do cenário dos RS e propôs uma técnica híbrida que combinava aspectos da filtragem baseada em conhecimento e filtragem colaborativa para o desenvolvimento do EntreeC, um sistema de recomendação de restaurantes baseado no Entree. Alguns anos depois, em "Hybrid Web Recommender Systems”, [Burke 2007] traz uma nova análise do cenário de RS em técnicas híbridas voltadas para a Web. O objetivo é demonstrar que a combinação entre diferentes técnicas de filtragem é capaz de minimizar limitações que as abordagens trazem individualmente.

Em 2004, [Penha 2004] realizou um estudo intitulado "Inteligência Artificial em Dispositivos Móveis: Estado da arte”, onde é abordado um levantamento histórico e evolutivo acerca da Inteligência Artificial e dos dispositivos móveis, demonstrando como a união entre estas duas grandezas pode potencializar as funcionalidades dos dispositivos e a experiênica do usuário.

Já a proposta de [Goren-Bar and Kuflik 2004] parece ser bastante interessante. No trabalho "Don't Miss-R - Recommending Restaurants through an Adaptive Mobile System", os autores realizam um experimento com o objetivo de obter uma comparação entre dois sistemas de recomendação para usuários móveis, um adaptativo e um não adaptativo, com o propósito de avaliar as preferências dos usuários com respeito à adaptatividade do sistema.

Mais recentemente, [Mack 2010] realiza um estudo intitulado "Sistema de recomendação baseado na localização e perfil utilizando a plataforma android", onde são destacados aspectos acerca de serviços baseados em localização e sistemas de recomendação. Ao fim, é proposta uma aplicação de sistema de recomendação com técnicas de filtragem híbridas que seja capaz de permitir que o usuário, através de seu smartphone, possa descobrir a localização de um estabelecimento próximo que o mesmo deseja visitar.

Algumas aplicações otimizam seus serviços com base em informações acerca do contexto onde o usuário está inserido e até mesmo da estimativa de seu perfil, com o objetivo de auxiliar em seus processos de tomada de decisão. Exemplos a serem considerados são o serviço de pesquisa e visualização do Google Maps [Google Inc. 2005], que fornece opções de rotas rodoviárias otimizadas para o destino desejado pelo indivíduo. 
Na literatura discutida, poucos trabalhos se assemelham aos objetivos desta proposta. Dentre eles, é importante destacar [Burke 2002], que desenvolveu um RS Web para sugerir restaurantes baseando-se em preferências coletadas explicitamente do usuário. Além deste, [Goren-Bar and Kuflik 2004] desenvolveu um RS adaptativo também voltado para restaurantes, mas relacionado à recomendação de itens de cardápio. Entretanto, a maior contribuição do trabalho foi a realização de uma análise da melhor estratégia de $\mathrm{RS}$, entre a adaptativa e a não adaptativa.

Por fim, a proposta de [Mack 2010] foi a que se apresentou mais próxima da proposta desta pesquisa. Contudo, a proposta representa apenas um modelo que recomenda estabelecimentos de acordo com os critérios de localização e reputação. Não há avaliação de itens específicos destes estabelecimentos, que é onde concentra-se o objetivo desta pesquisa. O sistema proposto também não realiza estimativas de perfil ou padrões de consumo dos usuários.

O fato de não ser possível traçar um perfil acerca do usuário móvel também incide no impedimento da personalização de ofertas através de anúncios personalizados. Estes, se realizados adequadamente, são importantes na perspectiva do crescimento do nível de satisfação do usuário. Também podem possibilitar, em momentos futuros, a monetização do sistema com um melhor custo-benefício, uma vez que investidores poderiam anunciar seus produtos e serviços de maneira direta para usuários específicos.

\section{Plataforma PGuide}

PGuide é uma plataforma que oferece um serviço de recomendação baseado em informações sobre o usuário e o contexto onde o mesmo está inserido. Seu objetivo principal é indicar sugestões de lugares e ofertas para itens que o usuário deseje com o melhor custo-benefício, oferecendo suporte para a tomada de decisão durante o processo de compras.

Sua funcionalidade primordial reside na possibilidade de realização de consultas e de recomendações de estabelecimentos comerciais que tenham disponíveis os itens que o indivíduo deseja. Ademais, o sistema permite a busca por ofertas de itens que são do interesse do usuário.

Para avaliar e classificar os estabelecimentos que possuem estes itens de acordo com o nível de interesse do usuário, a plataforma utiliza três grandezas básicas: preço do item, reputação obtida pelos estabelecimentos comerciais que o possuem através da avaliação de outros usuários, e distância para estes estabelecimentos. Já para a sugestão de ofertas, é necessária a análise do perfil do usuário para compreender quais itens são do interesse e quais não são. Para tal, o sistema conta com o acesso ao histórico de consultas do usuário.

Para ser capaz de executar estas funcionalidades, foi necessário definir uma arquitetura de software cliente-servidor, de modo que o cliente é representado pela aplicação móvel, executada em dispositivos tais como smartphones ou tablets $P C$ e denominada "PGuide Mobile", e o servidor é representado pela aplicação denominada "PGuide Server".

$\mathrm{Na}$ arquitetura proposta, ilustrada na Figura 2, os usuários móveis, através do PGuide Mobile, utilizam os recursos de rede disponíveis para acessar o servidor PGuide Server. Este, por sua vez, acessa suas bases de dados, que possuem informações acerca de itens que são de interesse do usuário, de reputação de estabelecimentos e sua localização 
geográfica, perfis de consumo de clientes, dentre outros.

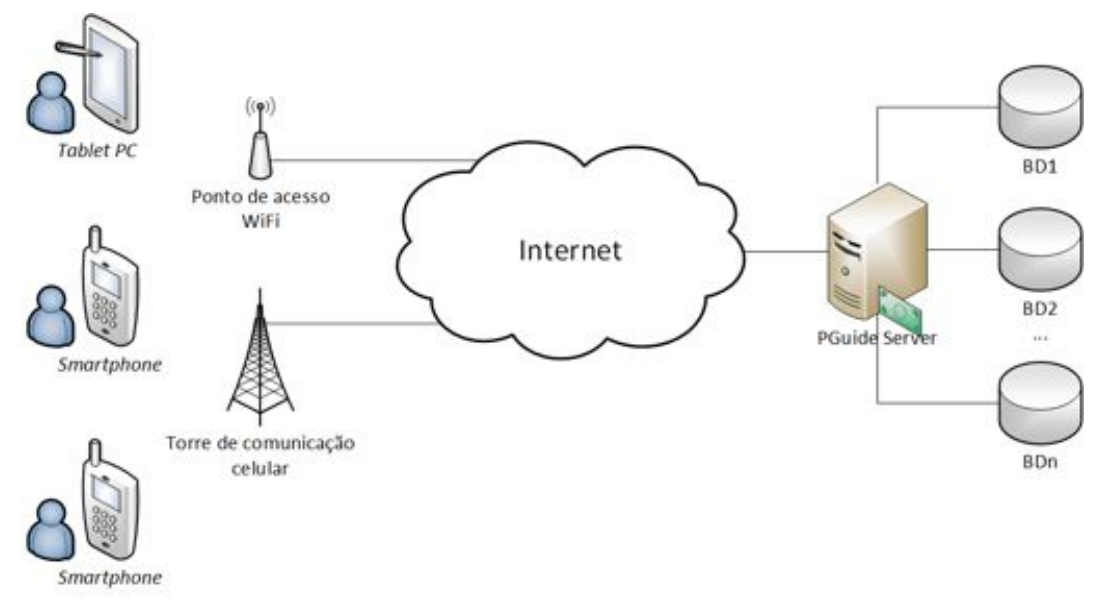

Figura 2. Arquitetura da Plataforma PGuide.

Em posse destes dados, os algoritmos de recomendação são capazes de executar cálculos para a realização de recomendações e retornar para o usuário as informações da forma que ele espera: uma lista ordenada com os lugares mais apropriados para adquirir os itens que ele escolheu, cada lugar associado a um índice de compatibilidade em relação aos interesses do usuário.

A aplicação móvel, em si, representa apenas um terminal de comunicação entre o usuário e o servidor (PGuide Server). Quando o usuário entra com dados ou dispara eventos na aplicação, a aplicação móvel envia solicitações para o servidor, que retorna o que lhe é solicitado: realização de autenticações, dados, listas de itens, entre outros. Esta comunicação é possível através da implementação serviços web (web services), que são implementados utilizando o protocolo JSON (JavaScript Object Notation).

A aplicação PGuide Server traz a principal contribuição deste trabalho. Ela armazena as fontes de dados utilizadas por toda a plataforma e executa o processamento dos modelos que permitem a recomendação de itens para o usuário.

Este conjunto (mobile e server) permite que a plataforma PGuide ofereça como solução, em seus algoritmos, a atribuição de pesos aos critérios que serão avaliados, de acordo com as preferências do usuário. Assim, o usuário tem a capacidade de manipular os pesos de cada um dos critérios de preferências com o objetivo de tornar os resultados cada vez mais compatíveis com seus interesses, variando de uma escala no intervalo de 0 (zero), que representa o menor peso, até 100 (cem), que representa o maior peso. A representação deste valor é denominada "índice de relevância".

Apesar de preço, distância e reputação serem grandezas distintas entre si, a classificação delas através de pesos possibilitará que elas associem-se entre si, convergindo para uma nova grandeza: a pontuação de compatibilidade que o item tem para com o usuário. Esta é calculada através da soma de três cálculos: pontuação de proximidade, pontuação de preço e pontuação por reputação. $\mathrm{O}$ cálculo destas pontuações obedece aos modelos apresentados a seguir.

A Fórmula 1 apresenta o cálculo de pontuação de proximidade $\left(P_{\text {proximidade }}\right)$. As variáveis $d_{\min }$ e $d_{\max }$ representam, respectivamente, os valores mínimo e máximo utiliza- 
dos como referência para o cálculo da pontuação referente à proximidade. Estes valores podem ser adquiridos de duas maneiras: a primeira é através do registro explícito informado pelo usuário, o que requer uma filtragem dos itens que obedecerão a este critério; a segunda é através de uma análise acerca dos itens que serão avaliados, onde o valor da distância do item que está localizado mais próximo ao usuário, assim como o mais distante, serão utilizados como referências nestes parâmetros. As variáveis $d$ e $i_{r}$ correspondem, respectivamente, à distância entre o usuário e o estabelecimento que possui o item a ser avaliado e o índice de relevância para o cálculo da pontuação de proximidade.

$$
P_{\text {proximidade }}=\frac{\left(100-\frac{\left(\left(d-d_{\min }\right) \times 100\right)}{d_{\max }-d_{\min }}\right) i_{r}}{100}
$$

O modelo realiza o cálculo da distância entre a localização atual do usuário móvel e o estabelecimento que possui o item a ser computado e, a partir deste resultado, calcula a pontuação através de uma regra de três simples que analisa o quão próximo dos valores mínimo e máximo a distância para o item analisado está. A este valor de pontuação, ao fim do algoritmo, aplica-se o valor do índice de relevância para este critério, de acordo com as preferências do usuário.

Já o modelo para o cálculo de pontuação de preço $\left(P_{\text {preco }}\right)$ é idêntico ao da proximidade, como descrita através da Fórmula 2, onde a variável $p$ representa o valor (preço) do item a ser analisado e as variáveis $p_{\min }$ e $p_{\max }$ representam, respectivamente, os valores mínimo e máximo para o critério "preço".

$$
P_{\text {preco }}=\frac{\left(100-\frac{\left(\left(p-p_{\min }\right) \times 100\right)}{p_{\max }-p_{\min }}\right) i_{r}}{100}
$$

Pode-se observar que, nos dois algoritmos apresentados, o valor da pontuação é resultante da subtração 100 - $X$, onde $X$ é o valor do cálculo da regra de três que envolve os valores máximos e mínimos para os critérios de distância e preço. Esta subtração justifica-se da seguinte forma: para os dois critérios (distância e preço), os valores que interessam ao usuário são os menores possíveis, ou seja, quanto mais baixo, mais útil. Dessa forma, os valores da proporção entre o item e os valores mínimo e máximo são dados numa escala que varia de 0 (mais barato ou mais próximo) a $100 \%$ (mais caro ou mais distante). Por isto, há a necessidade de ser realizada uma inversão destes valores. Para exemplificar, um item que possui o preço de $\mathrm{R} \$ 20$ numa escala onde o preço mínimo encontrado é $\mathrm{R} \$ 10$ e o máximo é $\mathrm{R} \$ 50$ atingirá uma pontuação de 25 pontos numa escala de 0 a 100 pontos. Portanto, com a inversão, percebe-se que a pontuação para este item será de $100-25=75 \%$ em relação aos outros itens.

O modelo para o cálculo de pontuação de reputação $\left(P_{\text {reputacao }}\right)$ é apresentado na Fórmula 3. Observa-se que o mesmo é similar ao modelo de cálculo da pontuação do preço, mas não segue a mesma linha dos algoritmos anteriores, onde o valor da pontuação é calculado com base na inversão do resultado. Isto ocorre porque o valor da reputação é proporcional à relação entre os valores mínimo e máximo. Ou seja, quanto maior o valor de reputação, mais indicado o item será para o usuário. Por isto, a inversão não se faz necessária nesta operação. 


$$
P_{\text {reputacao }}=\frac{\left(\frac{\left(r-r_{\min }\right) \times 100}{r_{\max }-r_{\min }}\right) i_{r}}{100}
$$

Para a realização da análise de uma variedade de ofertas de um item que é do interesse de um usuário, é estabelecida uma lista de estabelecimentos que possuem o item a ser avaliado. Para cada item desta lista, são calculadas as pontuações de distância, preço e reputação, onde a soma destas pontuações constitui a pontuação final do item, conforme mostrado na Fórmula 4. Ao fim, calculada a pontuação de cada estabelecimento, é elaborada uma lista ordenada de forma decrescente de acordo com a pontuação final $\left(P_{\text {final }}\right)$ de cada item. Esta é calculada de forma proporcional em relação à escala que varia de 0 a $100 \%$, tomando-se como limite máximo para o intervalo $\left(P_{\max }\right)$ o item que obteve a maior pontuação, na forma que apresenta a Fórmula 5. Assim, as maiores pontuações obtidas serão recomendadas com mais alto grau de compatibilidade com o usuário.

$$
\begin{gathered}
P_{\text {total }}=P_{\text {proximidade }}+P_{\text {preco }}+P_{\text {reputacao }} \\
P_{\text {final }}=\frac{100 \times P_{\text {total }}}{P_{\max }}
\end{gathered}
$$

Para a avaliação de uma lista de itens o processo é um pouco mais complexo. Foi admitido que, para que um estabelecimento seja avaliado, é necessário que ele possua todos os itens que foram adicionados à lista de interesses do usuário. Com isto, o critério preço agora não é mais avaliado em sua singularidade, mas em conjunto (somatório) com todos os outros itens e quantidades desejadas. Já os critérios distância e reputação são calculados da mesma forma que anteriormente.

$$
s_{\text {preco }}=\sum_{i=0}^{n}(p(i) \times q(i))
$$

Seja $n$ uma determinada quantidade de itens em uma lista de interesse do usuário, $p(i)$ e $q(i)$ o preço do item $i$ e a quantidade de unidades desejada deste mesmo item, respectivamente. $\mathrm{O}$ cálculo do preço total $\left(s_{\text {preco }}\right)$ se dá na forma apresentada na Fórmula 6. de itens de interesse do mesmo.

\section{Exemplo de aplicação}

Para o desenvolvimento da aplicação móvel PGuide foi escolhida a plataforma Android. Foram utilizadas duas instâncias para os testes durante o desenvolvimento: uma lógica, através do emulador disponibilizado com a $S D K$ do Android; e uma física, representada pelo dispositivo Samsung Galaxy Nexus. Já a aplicação servidor foi desenvolvida utilizando a linguagem de programação Python, através do framework Django.

A comunicação com o servidor é realizada através do módulo WebService, que utiliza o protocolo JSON. Seus métodos recebem as solicitações da aplicação e enviam 
mensagens ao servidor. Este analisa os dados recebidos na solicitação, realiza os procedimentos necessários (sejam de consulta, de registro ou de processamento) e retorna os dados através de pacotes JSON.

Entretanto, os dados retornados no formato JSON não são úteis à aplicação. É necessário realizar um processo de interpretação destes dados. Este papel é executado pelo módulo Parser, que recebe como entrada as respostas do web service e retorna no formato das estruturas de dados que a aplicação utiliza.

\subsection{Cenário de uso}

Para visualizar um exemplo de aplicabilidade da plataforma PGuide, considere o cenário fictício a seguir: um usuário realiza a busca pelo item "Álcool Etílico 500mL" da marca "Nobre". O mesmo está localizado na latitude -9.4154227 e longitude -36.6224096 e ajustou como prioridade nos critérios preço, distância e reputação o valor de $50 \%$ (ou seja, o usuário atribuiu o mesmo nível de prioridade para os três critérios). Considere também os dados sobre a disponibilidade do referido item nos estabelecimentos relativamente próximos, apresentado na Tabela 1.

Tabela 1. Preços do item "Álcool Etílico Hidratado", 500mL, marca "Nobre".

\begin{tabular}{|l|l|l|l|l|}
\hline Estabelecimento & Preço & Latitude & Longitude & Reputação \\
\hline Unicompra & $\mathrm{R} \$ 1,15$ & -9.408234 & -36.631316 & $4.6^{2}$ \\
\hline Todo Dia & $\mathrm{R} \$ 1,32$ & -9.409637 & -36.630286 & $4.0^{2}$ \\
\hline Mercadinho Vieira & $\mathrm{R} \$ 1,51$ & -9.414527 & -36.622379 & $3.5^{2}$ \\
\hline
\end{tabular}

Ao solicitar a realização de recomendação de estabelecimentos para o item selecionado, o usuário obteve as recomendações apresentadas na Figura 3 (esquerda).

Agora considere que o usuário, não satisfeito com os resultados, realizou uma alteração entre os pesos dos critérios de avaliação de preço, distância e reputação para $30 \%, 80 \%$ e $30 \%$, respectivamente. Assim, o critério que mais interessa ao usuário neste momento é uma distância mínima para se percorrer e chegar até o local desejado. Assim, foram observados os resultados apresentados na Figura 3 (direita).

Por fim, considerando os resultados satisfatórios, o usuário clica em uma das recomendações e, automaticamente, a aplicação inicia uma rota do Google Maps [Google Inc. 2005] com o percurso entre sua localização atual e a localização do estabelecimento escolhido. Isto é possível graças à integração através de uma chamada externa ao aplicativo Google Maps, que automatiza todo o procedimento de roteamento rodoviário, auxiliando o usuário a chegar até o seu destino.

\section{Considerações Finais}

Neste trabalho foi proposta a plataforma PGuide, ferramenta que utiliza técnicas de sistemas de recomendação para fornecer serviços voltados para o comércio eletrônico móvel. O mesmo foi importante por trazer uma análise sobre o estado da arte em técnicas de inteligência artificial, em especial as técnicas de sistemas de recomendação tradicionais e voltadas para serviços em dispositivos móveis.

\footnotetext{
${ }^{2} \mathrm{Na}$ plataforma PGuide, o valor da reputação de um estabelecimento varia entre 0 (zero) e 5 (cinco). Quando apresentado nas avaliações (recomendações) ao usuário, é representada numa escala que varia de 0 (zero) a 10 (dez).
} 


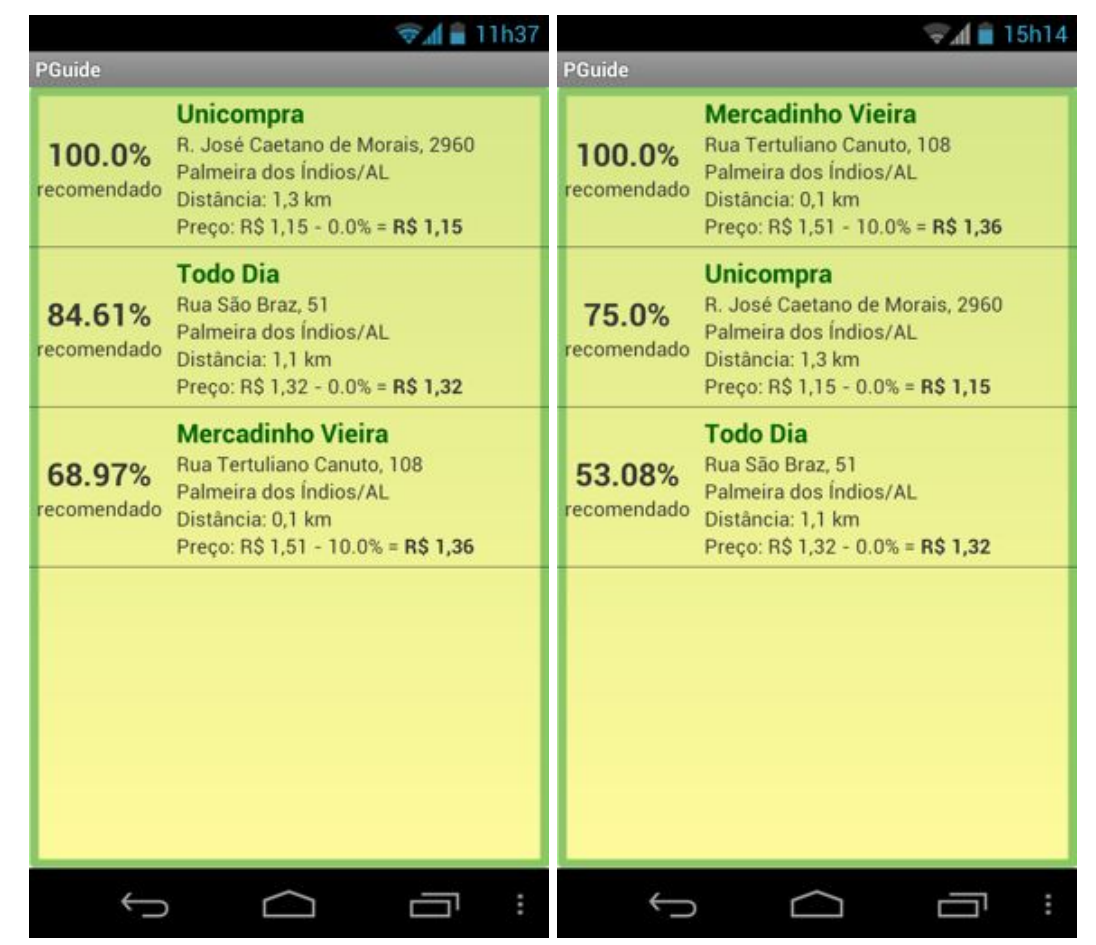

Figura 3. À esquerda, recomendação mesmo peso para todos os critérios de avaliação; à direita, recomendação para critérios com pesos diferentes.

A principal contribuição deste projeto reside nos modelos e algoritmos de recomendação de itens ao usuário que levam em consideração, simultaneamente, diversas grandezas que são do interesse do consumidor no comércio eletrônico, mesclando-as em um único parâmetro de valor percentual, com a finalidade de facilitar o processo de decisão do usuário.

Além disto, o exemplo de aplicação implementa protótipos da plataforma PGuide em duas arquiteturas: PGuide Mobile (aplicação para consumidores móveis) e PGuide Server (aplicação servidor que oferece o serviço).

Os resultados da pesquisa apresentada representam a concepção e introdução da plataforma PGuide. Assim, para a evolução deste estudo, muitas são as possibilidades de continuidade, expansão e implantação. Possíveis oportunidades de pesquisa podem ser vislumbradas, tais como:

- Realização da análise do desempenho dos algoritmos de recomendação e investigação de outras questões, tais como escalabilidade e disponibilidade, principalmente com a utilização de um maior volume de dados, inclusive dados reais, para obter um melhor processo de validação;

- Realização de testes aprofundados sobre os algoritmos de recomendação;

- Expansão dos algoritmos de recomendação, através da adição de outros critérios de avaliação e obtenção de pontuação, tais como formas de pagamento, condições climáticas ou de tráfego, análise de feedback, entre outros;

- Implementação de mecanismos de segurança e privacidade, visando oferecer maior confiabilidade do serviço para os usuários;

- Disponibilização do projeto na forma de um framework de recomendação. 


\section{Referências}

Burke, R. (2002). Hybrid Recommender Systems: Surveys and Experiments. User Modeling and User-Adapted Interaction (UMUAI)., pages 331-370.

Burke, R. (2007). Hybrid Web Recommender Systems. The Adaptative Web., pages 377-408.

Euzébio, M. V. M. (2011). DroidLar - Automação residencial através de um celular Android. Technical report, Instituto Federal de Santa Catarina.

Google Inc. (2005). Google maps. [online]; disponível em: http://maps.google.com em 27/10/2012.

Goren-Bar, D. and Kuflik, T. (2004). Don't Miss-R - Recommending Restaurants through an Adaptive Mobile System. In 9th International Conference on Intelligent User Interfaces, pages 250-252.

IDC (2012). Android- and iOS-Powered Smartphones Expand Their Share of the Market in the First Quarter, According to IDC. Technical report, International Data Corporation.

Lima, A. O., Ferreira, D. I. S., and de Sales, T. B. M. (2012). PGuide. In INOVA-BASE XII Escola Regional de Computação Bahia Alagoas Sergipe, Juazeiro-BA (Brasil).

Lops, P., de Gemmis, M., and Semeraro, G. (2011). Recommender Systems Handbook, chapter Content-based Recommender Systems: State of the Art and Trends. Springer.

Mack, R. S. (2010). Sistema de recomendação baseado na localização e perfil utilizando a plataforma android. Technical report, Universidade Federal do Rio Grande do Sul.

Mateus, G. R. and Loureiro, A. A. F. (2005). Indrodução à Computação Móvel. Departamento de Ciência da Computação da UFMG.

Nielsen Company (2011). The Mobile Media Report. State of the media - Q3/2011. Technical report, Nielsen.

Penha, G. B. (2004). Inteligência Artificial em dispositivos móveis. Technical report, Universidade Estadual de Londrina.

Resnick, P. and Varian, H. R. (1997). Recommender Systems. Communications of the ACM., 40(3):56-58.

Ricci, F. (2002). Travel Recommender Systems. IEEE Intelligent Systems., pages 55-57.

Ricci, F. (2010). Mobile Recommender Systems. International Journal of Information Technology and Tourism., pages 205-231.

Ricci, F., Shapira, B., Rokach, L., and Kantor, P. B. (2011). Recommender Systems Handbook, chapter Introduction to Recommender Systems Handbook. Springer.

Schafer, J. B., Konstan, J., and Riedl, J. (1999). Recommender Systems in E-Commerce. In 1st ACM conference on Electronic commerce, pages 158-166, USA.

Schafer, J. B., Konstan, J. A., and Riedl, J. (2001). E-Commerce Recommendation Applications. Data Mining and Knowledge Discovery., 5:115-153.

Weiser, M. (1991). The Computer for the 21st Century. Scientific American Ubicomp., pages 94-104. 